\title{
The effect of radiation velocity potentials on the drift force on a submerged sphere
}

\author{
S. H. Mousavizadegan \& M. Rahman \\ Department of Engineering Mathematics, Dalhousie University, Canada
}

\begin{abstract}
The effect of the motion of a submerged sphere on the horizontal drift force is investigated analytically. The multipole expansion method is used to derive analytical solutions for the diffraction and radiation velocity potentials in a series of associated Legendre functions. The second-order steady force is obtained by the far field method. The effect of all velocity potentials is taken into account in derivation of the horizontal drift force. The total contribution of the radiation velocity potential is minimal to the horizontal drift force if the center of mass is at a distance less than twenty percent of the radius from the center of the sphere. The effect of the radiation velocity potential in vicinity of the resonant frequency is augmented and may create a relatively large horizontal drift force.

Keywords: drift forces, radiation, diffraction, multipole expansion.
\end{abstract}

\section{Introduction}

Marine structures are usually designed to operate in a wave environment. Structural loading of the body surface under the water and unsteady motions of the body are two of the principal resulting problems. When the characteristic body dimension is comparable to the wave length, the potential effects dominate. The presence of the body alters the pattern of wave propagation in the vicinity of the structure and causes wave scattering. The body may also oscillate and cause the radiation of waves if the constraints are not sufficiently rigid. As a consequence, the body experiences reacting forces from the surrounding fluid and constraints. Due to the complexity of the associated boundary value problems with the wavebody interactions in the frame of the potential flow theory, analytical solutions can be obtained for a few special geometries. In general, a numerical solution of Laplace's equation along with the associated boundary conditions is imperative. 
The objective of this research paper is to obtain an analytical bench mark solution for the effect of the radiation velocity potential on the second-order horizontal drift force acting upon a submerged sphere in infinite fluid depth.

The problem of scattering of a surface wave by a fully submerged, rigid and stationary sphere was discussed by Gray [1]. Using the general solution of the Laplace equation in spherical coordinates, Gray [1] expressed the diffraction potential in a series solution of associated Legendre functions with unknown coefficients. Srokosz [4], in his study on a submerged sphere as an absorber of wave power, applied the multipole potentials derived by Thorne [5] to obtain the non-dimensional parameters of the surge and heave radiation problems. Wang [6] expressed the velocity potentials as a summation of a wave source at the center of a sphere with an infinite series of wave free potentials to discuss the free motions of a spherical submarine in infinite depth. Wu et al. [7] presented a solution for the drift forces along with the analysis of the exciting forces acting on a submerged sphere in finite water depth using the multipole expansion method. They applied both the far field and near field methods to obtain the drift forces. They only considered the contribution of the diffraction problem velocity potentials to the drift force. Rahman [3] studied the fields of the hydrodynamic diffraction pressure and fluid velocity around a submerged sphere in finite depth. He used the multipole expansion method to obtain the fluid velocity potential.

An analytical solution is obtained for the first-order problems and the horizontal drift force of a sphere in time-harmonic waves in fluid of infinite depth. The multipole expansion method is used to derive analytical solutions for the diffraction and radiation velocity potentials in a series of associated Legendre functions. The associated hydrodynamic coefficients are obtained, and the response amplitude operators of the surge, heave and pitch motions are computed, taking into account the effect of the position of the center of mass. The second-order steady force is obtained by the far field method. The effect of all velocity potentials is taken into account in derivation of the horizontal drift force. The results of the computations are presented in tabular form to provide a very precise benchmark solution to validate the numerical schemes.

\section{Formulation of the problem}

Two sets of coordinate systems are considered. One is a right-handed Cartesian coordinate system $(x, y, z)$ fixed in the fluid with $o z$ opposing the direction of gravity and $o-x y$ lying in the undisturbed free surface. The other set is the spherical coordinate system $(r, \theta, \psi)$ with the origin at the center of the sphere, as shown in Fig. 1.

It is considered that the amplitudes of both the incident wave and the motion of the sphere are small. Therefore, the usage of linearized potential theory is justified and the whole fluid flow can be characterized by a scalar function called the velocity potential. The symmetrical geometry of the sphere indicates that there are only three modes of motion responding to a disturbance in any given direction. These are the surge, heave and pitch motions, as the incident wave propagates 


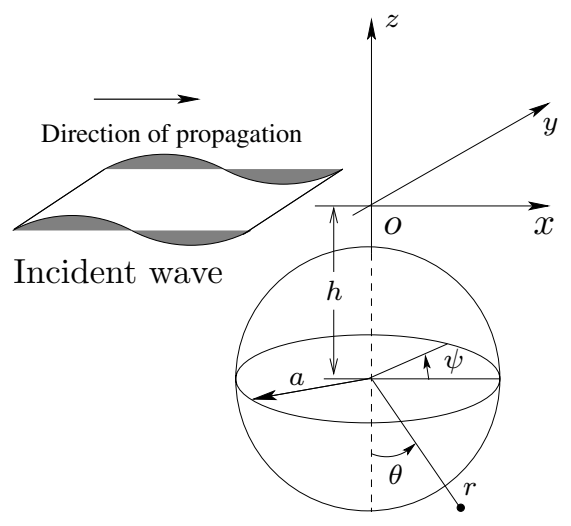

Figure 1: The sphere geometry and the coordinates definition.

along the $x$-axis. The hydrodynamic reactions in pitch motion vanish due to the symmetrical geometry of the sphere.

The total potential can be written as $\phi(r, \theta, \psi, t)=\left[\left(\eta_{1} \phi_{1}+\eta_{3} \phi_{3}\right)+A\left(\phi_{I}+\right.\right.$ $\left.\left.\phi_{S}\right)\right] e^{-i \omega t}$, where $\eta_{1}$ and $\eta_{3}$ are the amplitudes of the surge and heave motion, respectively, and $\phi_{1}$ and $\phi_{3}$ are the spatial velocity potential for the surge and heave motions, respectively. The notation $A$ is the amplitude of the incoming wave and $\phi_{I}$ and $\phi_{S}$ are the incoming and scattering wave time independent velocity potentials, respectively. The frequency of the incoming wave is given by $\omega$.

The radiation and scattering velocity potentials are subjected to the Laplace equation in the fluid domain, the linearized free surface boundary condition, a bottom boundary condition that indicates no flux through the bottom of the fluid, the radiation condition at infinity and the Neumann body surface boundary condition at the mean position of the body. The hydrodynamic forces and moments acting on the sphere due to the reaction of the fluid are obtained by the integration of the hydrodynamic pressure around the surface of the sphere.

The dynamic pressure due to the fluid velocity contributes to the second-order forces on the sphere, $P_{d}(r, \theta, \psi, t)=-\rho / 4 \Re\left[(\nabla \phi)^{2} e^{-i 2 \omega t}+\nabla \phi \nabla \phi^{*}\right]$. There is an oscillatory contribution and a time average part. The forces and moments resulting from the time average part are drift forces and moments, (for a more detail explanation refer to Mousavizadegan [2]).

\section{Drift force}

The sphere is also acted upon by a steady force in the direction of the incident wave propagation. The far field method is applied to find this component of the drift force. The total velocity potential $\Phi$ at large distance from the sphere may be 
defined by, Mousavizadegan [2]

$$
\Phi=\left\{-\frac{i g A}{\omega} e^{\nu z} e^{i \nu R \cos (\theta-\beta)}+\left(\frac{2}{\pi \nu R}\right)^{\frac{1}{2}} e^{\nu z} e^{i\left(\nu R+\frac{\pi}{4}\right)} H(\nu, \psi)\right\} e^{-i \omega t} .
$$

The main task is to obtain an expression for $H$-function to find the drift force. This function results from the scattering and radiating effects of the sphere in fluid domain. It may be written that, $H(\nu, \psi)=H_{S}(\nu, \psi)+H_{1}(\nu, \psi)+H_{3}(\nu, \psi)$, where $H_{S}(\nu, \psi)$ is the scattering $H$-function, $H_{1}(\nu, \psi)$ is the surge $H$-function and $H_{3}(\nu, \psi)$ is the heave $H$-function.

The scattering potential and the potentials due to the motion of the sphere in $x$ and $z$-directions can be expressed by

$$
\begin{gathered}
\phi_{S}(r, \theta, \psi)=\sum_{m=0}^{\infty} \sum_{n=m}^{\infty} a^{n+2} A_{n}^{m} \hat{\phi}_{n}^{m} \cos m \psi \text { and } \\
\phi_{j}^{m}(r, \theta, \psi)=\sum_{n=m}^{\infty} a^{n+2} B_{n}^{m} \hat{\phi_{n}^{m}} \cos m \psi,
\end{gathered}
$$

respectively, where $\hat{\phi}_{n}^{m}$ is the multipole potential. The coefficients $A_{n}^{m}$ and $B_{n}^{m}$ are obtained through the solution of the first-order problems of diffraction and radiation. These coefficient are computed by the solution of the following systems of linear algebraic equations.

$$
\begin{gathered}
\sum_{n=m}^{\infty} A_{n}^{m}\left[(n+1) P_{n}^{m}(\cos \theta)-\sum_{u=m}^{\infty}\left(C_{u n}^{m}+i D_{u n}^{m}\right) a^{n+u+1} P_{u}^{m}(\cos \theta)\right]= \\
A \omega e^{-\nu h} \varepsilon_{m} i^{m+1} \cos m \psi \sum_{u=m}^{\infty}(-1)^{m+u-1} \frac{u}{(u+m) !}(\nu a)^{u-1} P_{u}^{m}(\cos \theta) \\
\text { for } \quad m=0,1, \cdots \\
\sum_{n=1}^{\infty} B_{n}^{1}\left[(u+1) \delta_{u n}-\left(C_{u n}^{1}+i D_{u n}^{1}\right) u a^{n+u+1}\right]=-i \omega \delta_{u 1} \\
\sum_{n=0}^{\infty} B_{n}\left[(u+1) \delta_{u n}-\left(C_{u n}+i D_{u n}\right) s a^{u+s+1}\right]=i \omega \delta_{u 1} \\
\text { and } u=0,1,2, \cdots
\end{gathered}
$$

The coefficient $A_{n}^{m}$ is related to the diffraction problem, $B_{n}^{1}$ and $B_{n}$ are attributed to the radiation problem. The coefficient $B_{n}^{1}$ and $B_{n}$ are related to the surge and heave motions of the sphere, respectively. A detail description of the derivation of these system of linear algebraic equations and the definition of the attributed parameters are given in Mousavizadegan [2]. 
The asymptotic form of the multipole potential can be expressed according to Mousavizadegan [2] in the following form

$$
\hat{\phi}_{n}^{m} \rightarrow-2 \pi i \frac{(-1)^{m+n}}{(n-m) !} \nu^{n+1} e^{\nu(z-h)}\left(\frac{2}{\pi \nu R}\right)^{\frac{1}{2}} e^{i\left(\nu R-\frac{m \pi}{2}-\frac{\pi}{4}\right)}
$$

at a large distance from the sphere.

Substituting (5) in (2), the scattering and radiation velocity potentials are obtained and accordingly the $H$-functions are found in the form

$$
\begin{gathered}
H_{S}(\nu, \psi)=-2 \pi i e^{-\nu h} \sum_{m=0}^{\infty} \sum_{n=m}^{\infty} \frac{(-1)^{m+n}}{(n-m) !} a^{n+2} A_{n}^{m} \nu^{n+1} e^{-i\left(\frac{m+1}{2}\right) \pi} \cos m \psi \\
H_{1}(\nu, \psi)=2 \pi i e^{-\nu h} \sum_{n=1}^{\infty} \frac{(-1)^{n+1}}{(n-1) !} a^{n+2} B_{n}^{1} \nu^{n+1} \cos \psi \\
H_{3}(\nu, \psi)=-2 \pi e^{-\nu h} \sum_{n=0}^{\infty} \frac{(-1)^{n}}{n !} a^{n+2} B_{n} \nu^{n+1}
\end{gathered}
$$

where the $H_{1}(\nu, \psi)$ and $H_{3}(\nu, \psi)$ are for unit motion of the sphere in $x$ - and $z$ directions.

The horizontal drift forces and moment are obtained according to Mousavizadegan [2] by

$$
\begin{gathered}
\bar{F}_{x}=-\frac{\rho}{2 \pi} \int_{0}^{2 \pi} H(\nu, \theta) H^{*}(\nu, \theta) \cos \theta d \theta+\frac{\rho \omega A}{\nu} \Re[H(\nu, \beta)] \cos \beta \\
\bar{F}_{y}=-\frac{\rho}{2 \pi} \int_{0}^{2 \pi} H(\nu, \theta) H^{*}(\nu, \theta) \sin \theta d \theta+\frac{\rho \omega A}{\nu} \Re[H(\nu, \beta)] \sin \beta \\
\bar{M}_{z}=-\frac{\rho}{2 \pi \nu} \Im\left[\int_{0}^{2 \pi} H^{*}(\nu, \theta) H^{\prime}(\nu, \theta) d \theta\right]-\frac{\rho g A}{\omega \nu} \Im\left[H^{\prime}(\nu, \theta)\right] .
\end{gathered}
$$

The horizontal force in $y$-direction and the yaw drift moment are zero due to the orthogonal property of cosine and sine functions and the direction of the incident wave propagation along $x$-direction. Replacing (6) in (7), the horizontal drift force 
acting on the sphere in the $x$-direction is given in the form

$$
\begin{aligned}
& \bar{F}_{x}=-2 \pi \rho e^{-\nu h}\left\{\sum_{m=0}^{\infty}\left[e^{-\nu h} w_{m} \Im\left(C_{m} C_{m+1}^{*}\right)+\frac{\omega A}{\nu} \Re\left(e^{-i \frac{m \pi}{2}} C_{m}\right)\right]\right. \\
& +e^{-\nu h} \Im\left[C_{R 1}\left(w_{0} C_{0}^{*}-w_{2} C_{2}^{*}\right)\right]+\frac{\omega A}{\nu} \Re\left(e^{-i \frac{\pi}{1.1}} C_{R 1}\right) \\
& -e^{-\nu h} w_{0} \Im\left(C_{R 3} C_{1}^{*}\right)+\frac{\omega A}{\nu} \Re\left(C_{R 3}\right) \\
& \left.+e^{-\nu h} w_{0} \Im\left(C_{R 1} C_{R 3}^{*}\right)\right\}
\end{aligned}
$$

where

$$
\begin{aligned}
C_{m} & =\sum_{n=m}^{\infty} \frac{(-1)^{m+n}}{(n-m) !} a^{n+2} A_{n}^{m} \nu^{n+1}, \\
C_{R 1} & =\frac{\eta_{1}}{A} \sum_{n=1}^{\infty} \frac{(-1)^{n+1}}{(n-1) !} a^{n+2} B_{n}^{1} \nu^{n+1}, \\
C_{R 3} & =\frac{\eta_{3}}{A} \sum_{n=0}^{\infty} \frac{(-1)^{n}}{n !} a^{n+2} B_{n} \nu^{n+1},
\end{aligned}
$$

$w_{0}=2 \pi$ and $w_{m}=\pi$ for $m>0$. The parameters denoted by $\eta_{1} / A$ and $\eta_{3} / A$ are the non-dimensional amplitude of the surge and heave motion of the submerged sphere, respectively. These are obtained by the solution of the equations of the motions of the sphere. The equations of motions and a detail description of the computation of $\eta_{1} / A$ and $\eta_{3} / A$ can be found in Mousavizadegan [2].

The first bracket in (8) under the summation sign is due to the components of the diffraction potential. The first part in this bracket results from the scattering potential, and the second part is due to cross effect of the incoming and scattering waves. The second line in (8) shows the effect of the surge motion potential together with the scattering and the incident wave potentials. The third line is due to the effect of the heave potential together with the components of diffraction potential. The last term is due to the cross effect of the surge and heave potentials.

\section{Results and discussion}

The calculations were performed on a sphere of radius $a$ at different immersion depths. The immersion depth is the distance from the undisturbed free surface to the center of the sphere and is denoted by $h$. The computations are carried out where $h / a=1.25,1.5$ and 1.75 for a set of diffraction parameters in the range $0 \leq \nu a \leq 5$.

It is necessary to compute the coefficients $A_{n}^{m}$ for different values of $m$ and $n$. The infinite system of equations (3) are truncated to compute the coefficients $A_{n}^{m}$. It is considered that $m=0,1,2, \cdots, M$. For each system of equations $(\mathrm{m}=$ constant), the value of $n$ is set to be $n=m, m+1, \ldots, N$. A value of $N=18$ 
gives a set of stable solutions for $K a \leq 10$. These solutions do not change with an increase in the value of $N$. The number of systems of equations is set to be $M=11$ in computation of the horizontal drift force. This provides a set of stable solutions for the associated hydrodynamic coefficients with six decimal points for $0 \leq K a \leq 10$.

The coefficients $B_{n}$ and $B_{n}^{1}$ are obtained by solving the infinite systems of equations (4). The coefficient $B_{n}$ is related to the motion of the sphere along vertical axis $z$. The coefficient $B_{n}^{1}$ pertains to the oscillatory motion of the sphere in $x$-direction. These infinite systems of equations should also be truncated to find $B_{n}$ and $B_{n}^{1}$. The value of $n$ is set to be $n=m, m+1, \ldots, N$, where $m=0$ for the heave motion and $m=1$ for the surge motion. A set of stable solutions are obtained with six decimal points by assigning a value of $N=18$ for both coefficients concerned with the heave and surge motions of the sphere.

Table 1: The non-dimensional horizontal drift force due to the diffraction and radiation velocity potentials at $Z_{G} / a=0.1$.

\begin{tabular}{|c|ccccccc|}
\hline & \multicolumn{2}{|c}{ Diffraction problem effect } & \multicolumn{3}{c|}{ Radiation problem effect } \\
\hline$a$ & 1.25 & 1.5 & 1.75 & $h / a$ & 1.25 & 1.5 & 1.75 \\
\hline 0.0 & 0.000000 & 0.000000 & 0.000000 & 0.000000 & 0.000000 & 0.000000 \\
0.1 & 0.000074 & 0.000061 & 0.000053 & 0.000055 & 0.000051 & 0.000046 \\
0.2 & 0.001576 & 0.001145 & 0.000885 & 0.000703 & 0.000622 & 0.000522 \\
0.5 & 0.047610 & 0.022419 & 0.012160 & 0.021574 & 0.014924 & 0.009263 \\
1.0 & 0.166323 & 0.045473 & 0.015253 & 0.036821 & 0.013902 & 0.005112 \\
2.0 & 0.096621 & 0.012436 & 0.001786 & 0.027197 & -0.000210 & -0.000230 \\
3.0 & 0.036705 & 0.002088 & 0.000117 & 0.004178 & -0.000007 & -0.000009 \\
4.0 & 0.013933 & 0.000320 & 0.000006 & 0.000396 & 0.000002 & 0.000000 \\
5.0 & 0.005346 & 0.000046 & 0.000000 & 0.000021 & 0.000000 & 0.000000 \\
\hline
\end{tabular}

The horizontal drift force is computed using (8). In this equation, the first bracket under the summation sign is the contribution of the components of the diffraction velocity potential. The rest are the effect of the radiation velocity potentials. The drift force is made non-dimensional by dividing by $\frac{1}{2} \rho g A^{2} a$. Table 1 shows the calculated values for the non-dimensional horizontal drift force acting on a sphere, due to the effect of the components of the diffraction potential and the radiation potentials, separately.

The contribution of the diffraction velocity potential components to the horizontal drift force is more pronounced than the contribution of the radiation velocity potentials. The effect of the radiation velocity potentials to the horizontal drift force arises from their cross product either with each other or with the 


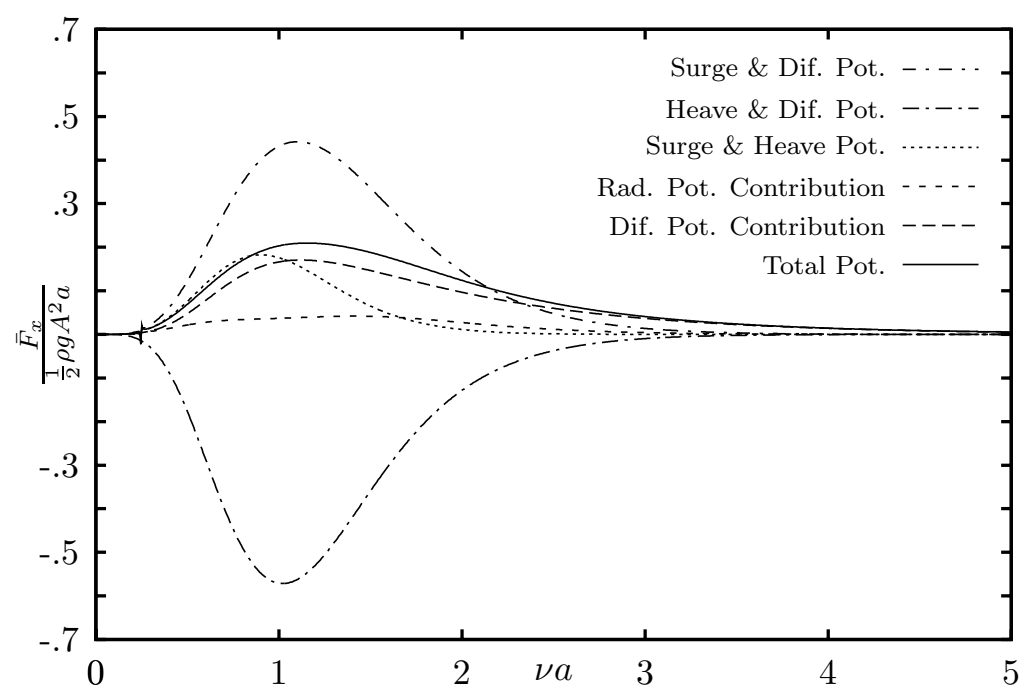

Figure 2: The effect of different velocity potentials on the horizontal drift force at $h / a=1.25$ and $Z_{G} / a=0.1$.

components of the diffraction velocity potential. The contribution of the diffraction problem velocity potentials to the horizontal drift force is about four times the effect of the radiation potentials in $0.7 \leq \nu a \leq 2.5$ at $h / a=1.25$ and $Z_{G} / a=0.1$. The effect of the radiation velocity potentials increases for $\nu a<0.7$, but is less than the effect of the diffraction potential components at $h / a=1.25$ and $Z_{G} / a=0.1$. The influence of the radiation velocity potentials is impaired for $\nu a>2.5$. The contribution of the radiation potentials decreases with increasing immersion depth.

The effect of each velocity potential on the horizontal drift force is depicted in Fig. 2 at $h / a=1.25$ and $Z_{G} / a=0.1$. The influence of the motion of the sphere may be separated into the cross effect resulting from the surge motion with the scattering and incoming waves, the contribution of heave motion with the scattering and incident waves and the surge motion with the heave motion. As illustrated in Fig. 2, the effect of the heave motion is almost canceled out by the effect of the two other components. Therefore, the contribution of the motion of the sphere is much less than the effect of the components of the diffraction velocity potentials.

The horizontal drift force is also affected by the position of the center of mass, as illustrated in Fig. 3. The ratio of the center of the mass to the radius of the sphere is set to vary from 0.1 to 0.5 . The sphere is more stable when $Z_{G} / a$ is increased. As shown in Fig. 3, a more stable sphere experiences a higher drift force. The effect of the radiation velocity potentials near the resonance frequency is significant. It may cause a rapid movement of the sphere near the resonance frequency. This is more 


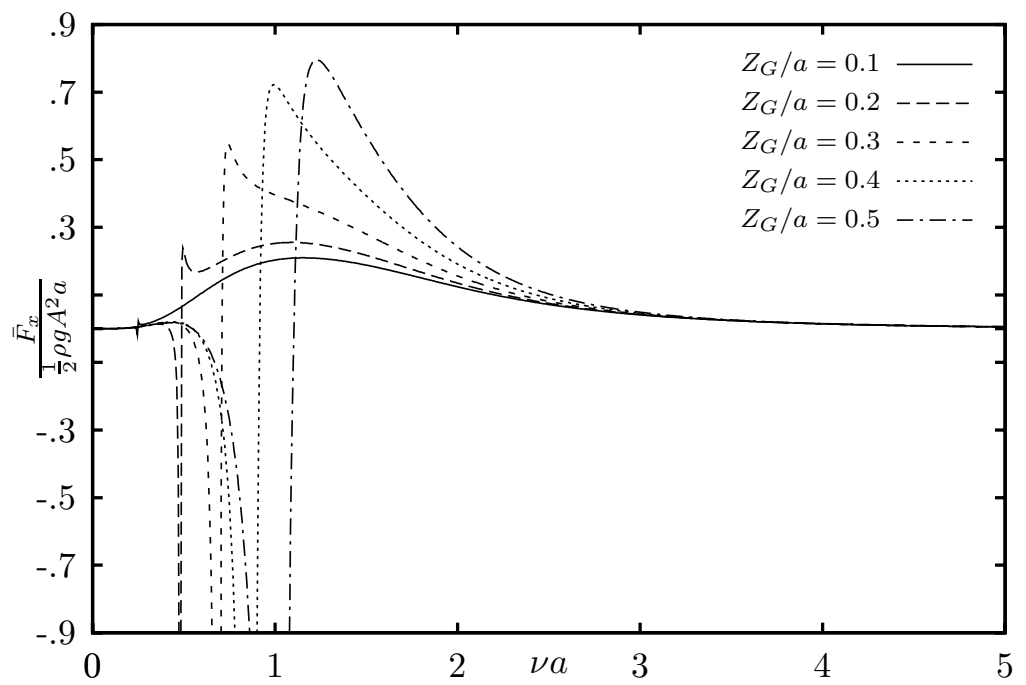

Figure 3: The effect of the location of the center mass on the horizontal drift force of a sphere at $h / a=1.25$.

pronounced when the stability of the sphere is increased. A wider range of wave spectrum is affected by the resonance frequency if $Z_{G} / a$ is increased. It seems that the proper position of the center of mass for a spherical structure is less than $0.2 a$ in respect of the horizontal drift force.

\section{Conclusions}

The results of the analytical solutions for a submerged sphere indicate that:

- the horizontal drift force acting on the spherical structure is mostly due to the effect of the components of the diffraction velocity potential;

- the individual components of the radiation velocity potential alone have no effect on the horizontal drift force;

- the effect of components of the radiation velocity potential results from the cross-product either with each other or with the scattering and incoming velocity potentials;

- the effect of the heave velocity potential almost cancels out the contribution of the surge velocity potential;

- the total contribution of the radiation velocity potential is minimal to the horizontal drift force if the center of mass is at a distance less than twenty percent of the radius from the center of the sphere;

- the effect of the radiation velocity potential in vicinity of the resonant frequency is augmented and may create a relatively large steady force; and 
- it is desirable to set the center of mass at a distance less than twenty percent of the radius from the center of the sphere.

This problem can be extended to a more general case and to study the effect of fluid depth on the drift force, taking into account the contribution of the radiation velocity potentials. It can be more generalized to derive the total drift force using the near field method, which is the integration of the dynamic pressure stemming from the fluid velocity around the body surface. This also provides the solution for the vertical drift force which may be useful in the study of the motion of spherical structures under the free surface of a fluid.

\section{Acknowledgement}

We are very grateful to Natural Sciences and Engineering Research Council (NSERC) of Canada for its financial support leading to this paper.

\section{References}

[1] E. P. Gray, Scattering of a surface wave by a submerged sphere, Journal of Engineering Mathematics 12 (1978), no. 1, 15-41.

[2] S. H. Mousavizadegan, Analytical and numerical approaches to determine the second-order forces in wave-body interactions, $\mathrm{PhD}$ thesis, Dalhousie University, September 2005.

[3] M. Rahman, Simulation of diffraction of ocean waves by a submerged sphere in finite depth, Applied ocean research 23 (2001), 305-317.

[4] M. A. Srokosz, The submerged sphere as an absorber of wave power, J. Fluid Mech. 95 (1979), 717-741.

[5] R. C. Thorne, Multipole expansions in the theory of surface waves, Proc. Camb. Phil. Soc. 49 (1953), 707-716.

[6] S. Wang, Motions of a spherical submarine in waves, Ocean Engng. 13 (1986), no. 3, 249-271.

[7] G. X. Wu, J. A. Witz, Q. Ma, and D. T. Brown, Analysis of wave induced drift forces acting on a submerged sphere in finite water depth, Applied ocean research 16 (1994), 353-361. 